\title{
Application of Redecision Therapy in Executive Coaching Workshops: Part 1 - the Workshop
}

\author{
(C) 2014 Mil Rosseau, Rik Rosseau \& Mark Widdowson
}

\begin{abstract}
First in a series of three, this paper describes how the redecision approach (Goulding \& Goulding 1979) has been applied over many years within executive coaching workshops internationally. The potential controversy about using a therapeutic approach in a business context is addressed, participant profiles and leadership characteristics are described, the impact of the group environment is considered, and the links between working on 'problems' and Berne's (1961) stages of cure are explained. The stages of working are related to those described by Goulding \& Goulding (1979) and supplemented with material from McNeel (1999-2000) and Allen \& Allen (2002). This paper describes the interventions that are evaluated qualitatively by Widdowson \& Rosseau (2014) and that will be further evaluated quantitatively in the future.
\end{abstract}

\section{Key words}

Redecision therapy, executive coaching, stages of cure, business context, group working

\section{Introduction}

This is the first article in a series of three. The second article (Widdowson \& Rosseau 2014) presents the findings from a qualitative study which investigated the outcomes of this model, and includes a selection of quotations from participants which often confirm the lifechanging nature of the intervention. The forthcoming third article will present results from a quantitative study, using an adapted version of the Ryff Scales of Psychological Well-being (Ryff 1989), which investigates changes in psychological well-being amongst participants of the workshops.

These articles are based on our experiences during three decades of facilitating developmental workshops in different industries in Europe, the USA, Asia, Africa and the Middle East. Participants have ranged in occupation from boardroom-level executives to manual workers.

\section{Redecision therapy in business}

We acknowledge that the notion of using redecision therapy (Goulding \& Goulding 1979) in business contexts may be regarded as controversial within the transactional analysis community. However, having worked as a psychotherapist with individuals and groups for over 10 years, prior to 'converting' to a business consultant, I (MR) perceive the differences between social control, symptomatic relief, transference cure and script cure (Berne 1961). People do reach cure in a business environment and it is far more than just 'understanding what happened'. They do regress, change the root cause and leave the workshop with profound and sustainable redecisions.

From the world of sports and into the business world, there is an increasing awareness that results are influenced by what goes on 'between the ears'. Gallwey (2000) is well known for his concept formula of $P=P-I$, which stands for "performance equals potential minus interference" (p.18). This comes extremely close to the Goulding \& Goulding (1986) comment about their redecision approach about helping clients to take away the straitjackets they have put on themselves in the past.

We believe, and expect to demonstrate in the later parts of this paper, that business environments have tremendous potential to build a third story, honouring Berne's (1966) vision as he described it during an interview at his Carmel house: "My feeling is that Freud has set the foundation. I think that we've built something on the foundation. Sometime in the future, someone will build the third story. And I think the answer is in script . . If we can get in there and break that up, than we can 
really change a person's life course. Not only for the person, but also for his children ... . [and] future generations."

\section{Participant profiles}

Samuels et al (1968), writing of short term psychotherapy, provided profiles we can relate to our participants:

- people who perceive their concerns as quite circumscribed - at the start of the workshops, participants have instrumental questions, such as how to improve relationships with colleagues, or be better negotiators, or deal with resistance during presentations;

- people who wish to sample counselling without commitment - in line with Berne's (1961) stages of cure, at first some participants want only social control over their behaviour; some may also symptomatic relief. During the workshop they discover and understand the underlying script system (Erskine \& Zalcman 1979) and move to the next stage: transference cure. At the end of the workshop, they create and refine an action plan and at the follow-up session many of them make statements such as "I was thinking of what you said, when I..." which indicates transference cure. Many also report a change in feeling, acting and thinking that has generalised and extended beyond the original contract. They then report they did some self-reparenting (James 1974). Those terms are our description of the process and not the way the participants report back to us.

- $\quad$ people whose problems or preoccupations are in the mild-to-moderate range - within the organisations we work with, we do not often see severe or life threatening pathology. Even with severely traumatised people, the participants are typically functioning at a high level and therefore are less likely to have severe pathology.

- $\quad$ people who are open to focal work - which applies to many of our participants!

- $\quad$ people who are well motivated and ready for change - even participants who are sceptical at the start of the workshops start contributing fully once they see the potential benefits of the training.

The following anecdote illustrates the impact of the workshops. A blue-collar employee, making a presentation on the workshop to visiting managers from central office, was asked what he considered to be his own major change after this program. To their surprise, he replied" I stopped beating my wife." When probed about this, he added "I learned here to respect people and how to gain the respect of others. When I go back home there is no way I can forget that."

\section{Leadership characteristics}

Leiberman et al (1973) studied the effectiveness of group therapy in 17 different types of groups and found that redecision therapy led by Bob Goulding was by far the most effective. McNeel (1982) found evidence of significant change for participants in redecision workshops run by Bob and Mary Goulding; Bader (1982) also found significant change in five families participating in redecision therapy as compared to controls; Kadis \& McLendon (1981) likewise reported high levels of change within 59 families that experienced redecision therapy.

The leadership behaviours correlated with the best outcomes that were identified by Lieberman et al (1973) can be related to the workshops:

- they are moderate in their use of emotional stimulation, not seducing the clients with their charisma.

- $\quad$ they work with contracts, which automatically puts the participants in the foreground.

- $\quad$ they are high in caring - the Gouldings ate and played with the participants; in the workshops we stay with the group from the very beginning, and make sure that we know what is happening in the business.

- they offer explanation of what they observe and information on how to change - we believe that observation combined with clear direction for change is one of the strengths of transactional analysis.

- they are moderate with their use of structured exercises - this sometimes creates challenges for us so we emphasise that the workshop is co-created. In our experience, participants used to Western and African cultures adjust quite easily whereas we notice that those used to the cultures of the Middle East and Asia need more time to adjust to the process.

\section{The group environment}

Change processes can be facilitated easily in a group environment. People get feedback from their peers, they can experiment with new solutions in a safe environment and they have the support of the other team members. Although in recent years the business world has had a tendency to shift towards individual coaching of their leaders, in our experience working in a group environment is a lot more effective. In this environment it is relatively easy to build protection (Crossman 1966) for change, so that participants can test new approaches and have them validated/confronted by peers. This is an experience which is difficult to engineer in individual sessions.

Commonly cited concerns about reluctance to open up in front of colleagues, or the danger of sharing information, 
are unfounded. Participants report gaining much mutual understanding and a higher degree of acceptance of socalled 'strange behaviours'; they learn to understand what Kets De Vries (2006) proposes:

1. You can explain all of human behavioor, even when it seems irrational.

2. Much human mental activity happens 'outside of conscious awareness'.

3. How people handle and express emotion is central to who they are.

4. Human development is an inter- and intrapersonal process.

A relevant quote of one of the participants is: "The most difficult part was the fact of telling life stories and opening up on things that still hurt very much in front of other people who were work colleagues. But this was also the part that most helped in order to understand the reason behind things and also to build trust among us."

\section{Stages of Working}

Goulding \& Goulding (1979) typically had therapy clients choose a scene, or memory, that represents an early experience of the current problem. In the business environment, people come to the workshops with a clear idea of what they want to accomplish. When prompted, they readily connect this to situations with parental figures. They tend to be experienced in quality management approaches and hence are used to the logic of 'regressing' to the root cause of a problem; hence they rarely question their own regression to earlier life experiences.

We understand this process in terms of Berne's (1961) stages of cure:

- social control involves bringing dysfunctional behaviours under control

- symptomatic relief comes from having a good understanding of intrapersonal functioning; participants go on to report that they are handling situations effectively that previously would have strongly invited them into scripty behaviour

- transference cure comes when participants symbolically and internally substitute the trainer for their parents - as when they claim: "I remember you said..." - often attributing words to us that we did not say - they have created an internally adjusted parent

- $\quad$ script cure - when the person takes over for themself the intrapsychic role they had projected onto the trainer

We have analysed the format of our workshops from detailed process notes and audio and video recordings and found that the general structure of our workshops is broadly in line with the three stages described by
Goulding and Goulding (1979), supplemented with the two stages they described in an interview with Bader (Goulding \& Goulding 1986), so the five stages are:

1. contact - 'saying hello' to the group

2. contract negotiation - as a group and also to work on tangible problems

3. impasse clarification - emotions, behaviours and thinking patterns connected to the problem, and how these fit the script system (Erskine \& Zalcman 1979)

4. redecision - where they recognise that injunctions are believed falsehoods (McNeel 1999-2000)

5. protection - preparing to apply the new decision in real life, with maintenance planning and reintegration back into family, workgroups and community, including setting up positive reinforcement to support the new decision (Allen \& Allen 2002).

\section{What next}

Part 2 of this paper is appearing in the same issue of this journal (Widdowson \& Rosseau 2104) and describes a qualitative study of the changes made by participants as a result of attending the workshops. Thematic analysis indicates that the impact was significant.

Part 3 is being planned and will be a quantitative study, probably using an adaptation of Ryff's (1989) material on psychological well-being.

Mil Rosseau is a Certified Transactional Analyst (Educational) and a lecturer at Nyenrode Business University. He can be contacted onmil@autonoom.com. Further information about the workshops may be obtained directly from Mil.

Mil Rosseau and Rik Rosseau are consultants at Autonoom nv.

Mark Widdowson is a Teaching \& Supervising Transactional Analyst (Psychotherapy).

\section{References}

Allen, J. \& Allen, B. (2002) Redecision Therapy as Brief Therapy in Tudor, K. Ed. Transactional Analysis, London: Sage 83-98

Bader, E (1982) Redecision in family therapy: a study of change in an intensive family therapy workshop Transactional Analysis Journal 12:1 27-38

Berne, E (1961) Transactional Analysis in Psycho-therapy. New York: Grove Press

Berne, E (1966)

http://www.youtube.com/watch?v=UwkDASzw Zs accessed 23 Jan 2014 
Crossman, P (1966) Permission and Protection Transactional Analysis Bulletin 5:19, 152-154

Erskine, R \& Zalcman, M (1979) The racket system; a model for racket analysis Transactional Analysis Journal 9:1 51-59

Gallwey, T (2000) The Inner Game of Work, Canada: Random House

Goulding, M M \& Goulding, R L (1986) Redecision Therapy (video recording) San Francisco: International Transactional Analysis Association

Goulding, R L. \& Goulding M M. (1979) Changing Lives through Redecision Therapy. New York: Grove Press Inc.

James, M (1974) Self reparenting; theory and process Transactional Analysis Journal 4: 3, 32-39

Kadis, L \& McLendon, R (1981) Redecision family therapy: its use with intensive multiple family groups American Journal of Family Therapy 9:2 75-83
Kets De Vries, M, (2006) The Leader On The Couch Chichester: John Wiley \& Sons

Lieberman, M A, Yalom, I D \& Miles, M B (1973) Encounter Groups: First Facts New York: Basic Books

McNeel, J (1982) Redecisions in psychotherapy: A study of the effects of an intensive weekend group workshop Transactional Analysis Journal 12:1 10-26

McNeel, J (1999-2000) Redecision Therapy as a process of New Belief Acquisition Journal of Redecision Therapy

Ryff C D (1989): Happiness is everything, or is it? Explorations on the meaning of psychological wellbeing. Journal of Personality and Social Psychology, 57:1069-1081.

Samuels, S.D., Teutsch C.K. \& Everts, K (1968) Short Term Psychotherapy Transactional Analysis Bulletin, 7: 28 83-84

Widdowson, M \& Rosseau, M (2014) Application of Redecision Therapy in Executive Coaching Workshops: Part 2 - A qualitative exploration of participants' changes International Journal of Transactional Analysis Research 5:1 19-30 